LEMOS, M.A.; GAMA, E.E.G.; MENEZES, D.; SANTOS, V.F.; TABOSA, J.N. Avaliação de dez linhagens e seus híbridos de milho superdoce em um dialelo completo. Horticultura Brasileira, Brasília, v. 20, n. 2, p. 167-170, junho 2.002.

\title{
Avaliação de dez linhagens e seus híbridos de milho superdoce em um dialelo completo
}

\author{
Margarida Agostinho Lemos; ; Elto Eugênio G. Gama²; Dimas Menezes; Venézio Felipe dos Santos³; José \\ Nildo Tabosa ${ }^{3}$.

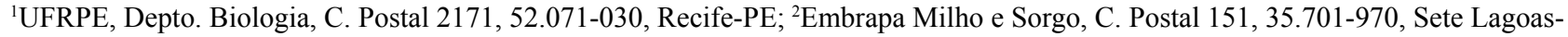 \\ MG; ${ }^{3}$ IPA, C. Postal 1022, 50.761-000, Recife-PE. E-mail: gamaelto@cnpms.embrapa.br
}

\section{RESUMO}

Em 1996/97 foram avaliados em Pesqueira e Vitória de Santo Antão (PE), 45 híbridos simples superdoce obtidos de um cruzamento dialélico completo entre dez linhagens $\mathrm{S}_{4}$ e suas linhagens parentais. O delineamento utilizado foi látice 7 × 8 com duas repetições por local. Foram estimados os efeitos das capacidades geral (CGC) e específica (CEC) de combinação e os valores de heterose para peso de espiga com palha (PECP), peso de espiga sem palha $(\mathrm{PESP})$ e rendimento de espiga $(\mathrm{REND}=\mathrm{PESP} / \mathrm{PECP} \times 100)$. Os efeitos de CGC e CEC foram significativos $(\mathrm{P}<0,01)$ para todos os caracteres. As linhagens com maiores REND foram $\mathrm{L}_{5}=76,5 \% ; \mathrm{L}_{7}$ $=71,2 \%$ e $\mathrm{L}_{4}=69,6 \%$ (Pesqueira); $\mathrm{L}_{4}=70,8 \% ; \mathrm{L}_{5}=69,4 \%$ e $\mathrm{L}_{7}=$ $65,3 \%$ (Vitória de Santo Antão), e estas também apresentaram os maiores valores para os efeitos da CGC nos dois locais. Os cruzamentos mais produtivos (REND) foram $\mathrm{L}_{4} \times \mathrm{L}_{8}=74,9 \%$ (Pesqueira) e $\mathrm{L}_{7} \times \mathrm{L}_{8}=70,3 \%$ (Vitória de Santo Antão), que também apresentaram os maiores efeitos da CEC nos dois locais. Para o caráter REND, os valores de heterose em relação à média dos pais variaram de $-20,19$ a 24,01\% (Pesqueira) e de - 18,02 a 16,76\% (Vitória de Santo Antão). Em relação ao pai mais produtivo os valores variaram de $-23,52$ a 22,99\% (Pesqueira) e de -22,05 a 12,03\% (Vitória de Santo Antão).

Palavras-chave: Zea mays L., capacidade combinatória, características agronômicas, heterose.

\begin{abstract}
Evaluation of ten inbred lines and their hybrids of supersweet maize in a complete diallel cross
\end{abstract}

In 1996/97, 45 single cross supersweet maize hybrids, from a complete diallel cross involving ten inbred lines $\left(\mathrm{S}_{4}\right)$ and their parents, were evaluated at Pesqueira and Vitória de Santo Antão, Brazil. A látice $7 \times 8$ design was used with two replications for each location. The general (GCA) and specific (SCA) combining ability effects and heterosis value were estimated for husked ear weight (HEW), unhusked ear weight (UEW) and ear rate $(E R=$ UEW/HEW x 100). The general and specific combining ability effects were significant $(\mathrm{P}<0.01)$ for all characteristics. Inbreds $\mathrm{L}_{5}=76.5 \% ; \mathrm{L}_{7}=71.2 \%$ and $\mathrm{L}_{4}=69.6 \%$ (Pesqueira); $\mathrm{L}_{4}=70.8 \% ; \mathrm{L}_{5}=69.4 \%$ and $\mathrm{L}_{7}=65.3 \%$ (Vitória de Santo Antão) showed the highest (ER), and the highest GCA effects at both locations. The best crosses for ER were: $\mathrm{L}_{4} \times \mathrm{L}_{8}$ $=74.9 \%$ (Pesqueira) and $\mathrm{L}_{7 \times} \mathrm{L}_{8}=70.3 \%$ (Vitória de Santo Antão), with the highest SCA effects at both locations. For ER, heterosis values related to parental mean ranged from -20.19 to $24.01 \%$ (Pesqueira) and -18.02 to $16.76 \%$ (Vitória de Santo Antão). Heterosis values related to the higher yielding parent ranged from -23.52 to $22.99 \%$ (Pesqueira) and -22.05 to $12.03 \%$ (Vitória de Santo Antão).

Keywords: Zea mays L., combining ability, agronomic traits, heterosis.

(Aceito para publicação em 06 de março de 2.002)

$\mathrm{M}^{\mathrm{i}}$ ilhos doces (Zea mays L.) são caracterizados por grãos com altos teores de açúcares e pouco amido no endosperma, o que os torna enrugados e translúcidos quando secos. A doçura é um caráter recessivo (Wallace \& Bressman, 1949) e os genes mutantes mais conhecidos são o sugary (su), shrunken (sh) e brittle (bt). Os genes bt e $s h$ acumulam açúcares às expensas de amido e apresentam teores muito baixos de carboidrato total no estágio de maturação. Devido aos elevados teores de açúcares, as cultivares dos genótipos $s h$ e $b t$ são chamadas de superdoces (Tracy, 1994).

O milho doce apresenta uma versatilidade de uso muito grande, além de agregar valor ao produto. Pode ser utilizado em conserva, enlatado, congelado na forma de espigas ou grãos, desidratado, consumido "in natura", colhido antes da polinização e usado como "Bay Corn" ou Mini-Milho e ainda após a colheita, a palhada da cultura pode ser utilizada para ensilagem (Souza et al., 1990).

No Brasil, algumas empresas governamentais e particulares vêm desenvolvendo programas de melhoramento para a produção de híbridos triplos de milho superdoce adaptados às principais áreas produtoras. Entretanto para as fábricas de processamento industrial de produtos vegetais localizadas na região Nordeste, ainda não existe material de valor comercial. Em vista disso, foi desenvolvida uma parceria entre a Embrapa, a Universidade Federal Ru- ral de Pernambuco, Fábricas Peixe e a Empresa Pernambucana de Pesquisa Agropecuária (IPA) na busca de material mais adaptado às condições do Estado de Pernambuco.

A obtenção de linhagem e o seu comportamento em combinações híbridas (capacidade de combinação), bem como o potencial "per se" é um dos objetivos básicos num programa de melhoramento genético pelo seu emprego na formação de híbridos comerciais. Sprague e Tatum (1942) refinaram o conceito de capacidade de combinação e propuseram os conceitos de capacidade geral e capacidade específica de combinação, relacionando-os, respectivamente, aos efeitos gênicos aditivos e não aditivos (dominantes e epistáticos). A 
obtenção dessas informações pode ser através de um método genético-estatístico que usa cruzamentos dialélicos. Estes cruzamentos compreendem um grupo de $\mathrm{p}$ linhagens autofecundadas, as quais são cruzadas entre si e fornecem um máximo de $\mathrm{p}^{2}$ combinações.

O sistema de cruzamento dialélicos se mostra bastante eficiente para avaliar as linhagens, pois, além de identificar os melhores híbridos simples, também auxilia na seleção daquelas mais promissoras para entrarem em um programa de melhoramento. Griffing (1956) desenvolveu uma metodologia de análise dialélica onde podem ser determinados os valores dos efeitos das capacidades geral e específica de combinação e heteroses, de acordo com a inclusão ou não dos pais e/ou híbridos $\mathrm{F}_{1}$ recíprocos. O Método 2, Modelo 1, apresentado por este autor, caracteriza-se pela inclusão dos materiais paternais e todos os híbridos $\mathrm{F}_{1}$ resultantes dos cruzamentos entre si, dois a dois, destes materiais, sem a inclusão dos recíprocos.

O objetivo deste trabalho foi avaliar as capacidades geral e específica de combinação e valores de heterose de dez linhagens de milho superdoce por meio de cruzamentos dialélicos, considerando três caracteres de espiga.

\section{MATERIAL E MÉTODOS}

O presente trabalho constou de dois experimentos. O primeiro, instalado em outubro/96, na Unidade das Fábricas Peixe, no município de Pesqueira (PE). $\mathrm{O}$ outro experimento foi instalado em agosto/97 no Campo Experimental de Vitória de Santo Antão (PE) da IPA.

Foram avaliados 56 tratamentos, sendo 10 linhagens e seus 45 híbridos simples e a cultivar Colorado DO2 usada como testemunha.

Essas linhagens superdoce $\left(\mathrm{S}_{4}\right)$ foram originadas a partir do cruzamento de linhagens elites normais de diferentes origens genéticas do programa de melhoramento da Embrapa Milho e Sorgo com uma fonte contendo o gene shrunken-2. Foram elas: LCMS2201 (L $)$, LCMS2202 $\left(\mathrm{L}_{2}\right)$, LCMS2203 $\left(\mathrm{L}_{3}\right)$, LCMS2204 $\left(\mathrm{L}_{4}\right)$, LCMS2205 ( $\left.\mathrm{L}_{5}\right)$, LCMS2206 ( $\left.\mathrm{L}_{6}\right)$, LCMS2207 ( $\left.\mathrm{L}_{7}\right)$, LCMS2208 $\left(\mathrm{L}_{8}\right)$, LCMS2209 $\left(\mathrm{L}_{9}\right)$ e LCMS2210 $\left(\mathrm{L}_{10}\right)$.
O delineamento experimental utilizado foi látice simples $7 \times 8$ (Cochran \& Cox, 1957) com duas repetições por local. O espaçamento utilizado foi 0,90 $\mathrm{m}$ entre fileiras e $0,20 \mathrm{~m}$ entre plantas dentro da fileira; a unidade experimental foi constituída por uma fileira de 5 $\mathrm{m}$ de comprimento. Foi deixada uma planta por cova após o desbaste. A adubação foi feita de acordo com a recomendação da análise de solo e foram incorporadas 15 toneladas de esterco de curral por hectare. Foi utilizada irrigação por aspersão sempre que necessário. Foram avaliados os caracteres peso de espigas com palha (PECP), peso de espigas sem palha (PESP) e rendimento de espiga $($ REND $=$ PESP/PECP $x$ 100). Para análise da capacidade combinatória, foi utilizado o Método 2, Modelo 1, de Griffing (1956). Foram estimados os efeitos das capacidades geral e específica de combinação. A determinação dos valores da heterose foi realizada através da comparação de cada híbrido com o valor da média dos pais e com o valor do pai mais produtivo.

\section{RESULTADOS E DISCUSSÃO}

$\mathrm{Na}$ análise de variância conjunta para peso de espiga com palha (PECP), peso de espiga sem palha (PESP) e rendimento de espiga (REND) o teste $\mathrm{F}$ indicou diferenças significativas entre linhagens $(\mathrm{P}<0,01)$ para os três caracteres. Os híbridos simples tiveram comportamento diferenciado para PESP $(\mathrm{P}<0,05)$, para PECP e REND $(\mathrm{P}<0,01)$. Estes resultados evidenciaram que as variações foram devidas, principalmente, a diferenças predominantemente genotípicas. Os efeitos para capacidade geral de combinação (CGC) e capacidade específica de combinação (CEC) foram também significativos $(\mathrm{P}<0,01)$, indicando a existência de variabilidade originada de efeitos gênicos aditivos e não aditivos. Resultados semelhantes foram encontrados por Parentoni et al. (1991), em trabalho com cruzamentos dialélicos entre dez linhagens de milho doce. O quadrado médio da CGC foi bem superior ao da CEC para os caracteres PECP e REND, indicado que os efeitos gênicos aditivos poderão ser mais importantes na expressão desses caracteres. Analisando os desvios-pa- drão dos caracteres (estimados conforme Vencosvsky, 1969), verificou-se maior variação dos efeitos da CEC. Essa maior variação já era esperada, uma vez que, para linhagens de origens mais homogêneas, em geral a CEC é mais efetiva que a CGC (Allard, 1960; Sprague \& Tatum, 1942).

Os genótipos não produzem efeitos constantes em cada ambiente, já que podem reagir de modo distinto a condições externas diferentes (Gnoatto, 1969). Por esta razão destaca-se, mediante análise conjunta, a sensibilidade dos parâmetros com os locais. Neste estudo, a interação linhagens versus local foi significativa para PECP $(\mathrm{P}<0,01)$ enquanto que, para híbridos versus local houve significância para PESP e PECP $(\mathrm{P}<0,05)$.

Os valores do teste $\mathrm{F}$ para a interação CGC x locais foram também significativos $(\mathrm{P}<0,01)$ para PECP e PESP. Já para a interação CEC x locais, os valores mostraram significância para PESP $(\mathrm{P}<0,05)$ e $\mathrm{REND}(\mathrm{P}<0,01)$. Isso evidencia que, para esses caracteres, as estimativas da capacidade combinatória não são consistentes com a variação ambiental e, por isso, na situação onde ocorreu interação, os efeitos de CGC e CEC devem ser estimados com base nas médias de cada local. Resultados semelhantes foram obtidos por Lima et al. (1995), Lopes et al. (1985) e Naspolini et al. (1981).

De acordo com Sprague e Tatum (1942), baixas estimativas de $\mathrm{G}_{\mathrm{i}}$ indicam genótipos com combinações que não diferem muito da média de todos os cruzamentos no sistema dialélico, enquanto altos valores (com sinal positivo ou negativo) indicam genótipos melhores ou piores que os restantes, com os quais se compara, e estes valores indicam, portanto, a importância dos genes de efeitos predominantemente aditivos. Em relação ao comportamento das linhagens (Tabela 1) observou-se que, para PECP, os maiores $\mathrm{G}_{\mathrm{i}}$ foram constatados para as linhagens $\mathrm{L}_{1}$ e $\mathrm{L}_{2}$, em Pesqueira, e $\mathrm{L}_{9}$ e $\mathrm{L}_{2}$, em Vitória de Santo Antão. Os maiores valores para o caráter PESP foram encontrados para as linhagens $\mathrm{L}_{4}$ e $\mathrm{L}_{1}$, em Pesqueira, e $\mathrm{L}_{9}$ e $\mathrm{L}_{6}$, em Vitória de Santo Antão. Considerando REND, foi verificado que as melhores linhagens 
Tabela 1. Estimativas dos efeitos da capacidade geral de combinação $\left(G_{i}\right)$ e médias de peso de espiga com palha (PECP), peso de espiga sem palha (PESP) e rendimento de espiga (REND), envolvendo dez linhagens de milho superdoce. Pesqueira e Vitória de Santo Antão (PE), UFRPE/IPA, 1996/97.

\begin{tabular}{|c|c|c|c|c|c|c|c|c|c|c|c|c|}
\hline \multirow{3}{*}{$\begin{array}{c}\text { Linha-- } \\
\text { gens }\end{array}$} & \multicolumn{4}{|c|}{ PECP } & \multicolumn{4}{|c|}{ PESP } & \multicolumn{4}{|c|}{ REND } \\
\hline & \multicolumn{2}{|c|}{ Pesqueira } & \multicolumn{2}{|c|}{$\begin{array}{c}\text { Vitória de Santo } \\
\text { Antão }\end{array}$} & \multicolumn{2}{|c|}{ Pesqueira } & \multicolumn{2}{|c|}{$\begin{array}{c}\text { Vitória de Santo } \\
\text { Antão }\end{array}$} & \multicolumn{2}{|c|}{ Pesqueira } & \multicolumn{2}{|c|}{$\begin{array}{c}\text { Vitória de Santo } \\
\text { Antão }\end{array}$} \\
\hline & $\mathbf{G i}$ & (g/planta) & $\mathbf{G i}$ & $\begin{array}{c}\text { (g/ } \\
\text { planta) }\end{array}$ & $\mathbf{G i}$ & $\begin{array}{c}\text { (g/ } \\
\text { planta) }\end{array}$ & $\mathbf{G i}$ & $\begin{array}{c}\text { (g/ } \\
\text { planta) }\end{array}$ & $\mathbf{G i}$ & $\begin{array}{c}\text { (Pesp/ } \\
\text { pecpx100) }\end{array}$ & $\mathbf{G i}$ & $\begin{array}{c}\text { (Pesp/ } \\
\text { pecpx100) }\end{array}$ \\
\hline$\overline{\mathrm{L}_{1}}$ & 20,502 & $211,6 b \ldots e$ & $-45,393$ & $163,8 \mathrm{e}$ & 9,797 & $114,8 \mathrm{bc}$ & $-36,103$ & $84,3 d$ & $-0,013$ & $54,3 d$ & $-0,032$ & $51,5 \mathrm{e}$ \\
\hline $\mathrm{L}_{2}$ & 16,735 & $299,0 a$ & 46,173 & $463,8 a b$ & 0,788 & $172,6 a b$ & 12,580 & $282,2 a b$ & $-0,034$ & $57,7 \mathrm{~cd}$ & $-0,045$ & $60,9 \mathrm{bcd}$ \\
\hline $\mathrm{L}_{3}$ & $-7,390$ & 181,4 de & $-36,785$ & 276,0 de & $-1,170$ & $112,9 \mathrm{bc}$ & $-26,953$ & $159,4 \mathrm{c}$ & 0,012 & $62,2 \mathrm{bcd}$ & $-0,001$ & $57,8 \mathrm{~cd}$ \\
\hline $\mathrm{L}_{4}$ & 5,085 & $295,1 \mathrm{ab}$ & $-22,652$ & $325,0 \mathrm{~cd}$ & 11,938 & $205,3 a$ & 1,622 & $230,2 b c$ & 0,031 & $69,6 a b$ & 0,036 & $70,8 a$ \\
\hline $\mathrm{L}_{5}$ & $-17,123$ & 207,4 cde & 4,673 & $480,6 a b$ & $-8,612$ & $158,7 a b$ & 14,555 & $333,5 a$ & 0,019 & $76,5 a$ & 0,035 & $69,4 a$ \\
\hline $\mathrm{L}_{6}$ & $-2,232$ & $303,3 a$ & 12,973 & $378,6 \mathrm{bcd}$ & 3,980 & $207,3 a$ & 15,472 & $238,1 \mathrm{bc}$ & 0,014 & $68,4 a b c$ & 0,010 & $62,9 \mathrm{bcd}$ \\
\hline $\mathrm{L}_{7}$ & $-7,390$ & $228,7 a \ldots e$ & $-2,610$ & $352,7 \mathrm{bcd}$ & 2,397 & $162,9 a b$ & 13,330 & $230,4 \mathrm{bc}$ & 0,022 & $71,2 a b c$ & 0,034 & $65,3 a b$ \\
\hline $\mathrm{L}_{8}$ & 4,910 & $146,0 \mathrm{e}$ & $-6,743$ & $345,7 \mathrm{bcd}$ & 1,963 & $80,0 c$ & $-9,403$ & $223,6 \mathrm{bc}$ & $-0,012$ & $54,8 d$ & $-0,008$ & $64,7 \mathrm{bc}$ \\
\hline $\mathrm{L}_{9}$ & $-6,965$ & $256,3 a \ldots d$ & 58,815 & $571,4 a$ & $-11,070$ & $152,6 a b$ & 17,705 & $295,1 a b$ & $-0,021$ & $59,5 \mathrm{bcd}$ & $-0,033$ & $51,5 \mathrm{de}$ \\
\hline$L_{10}$ & $-6,132$ & $267,6 \mathrm{abc}$ & $-8,452$ & $430,4 a b c$ & $-10,012$ & $160,8 a b$ & $-2,803$ & $267,1 \mathrm{ab}$ & $-0,019$ & $60,1 \mathrm{bcd}$ & $-0,004$ & $62,0 \mathrm{bcd}$ \\
\hline Média & & 239,6 & & 378,8 & & 152,8 & & 234,4 & & 63,4 & & 61,7 \\
\hline$\overline{C . V(\%)}$ & & 11,47 & & 15,00 & & 14,34 & & 14,34 & & 7,23 & & 4,22 \\
\hline
\end{tabular}

Médias seguidas de mesma letra nas colunas não diferem estatisticamente entre si a 5\% de probabilidade pelo teste de Duncan.

para os dois locais foram $\mathrm{L}_{4}, \mathrm{~L}_{5}$ e $\mathrm{L}_{7}$. Estas, por apresentarem mais alta concentração de alelos favoráveis para incremento do caráter, poderão mostrar médias dos cruzamentos em que participam, superiores à média geral de todos os híbridos simples. Por outro lado, as linhagens $\mathrm{L}_{5}$ e $\mathrm{L}_{3}$, em Pesqueira, e $\mathrm{L}_{1}$ e $\mathrm{L}_{3}$, em Vitória de Santo Antão, apresentaram as maiores magnitudes de efeitos negativos para PECP. Para PESP, foi constatado que as linhagens $\mathrm{L}_{9}$ e $\mathrm{L}_{10} \mathrm{em}$ Pesqueira e $\mathrm{L}_{1}$ e $\mathrm{L}_{3}$ em Vitória de Santo Antão apresentaram os maiores efeitos negativos, bem como as linhagens $\mathrm{L}_{2} \mathrm{e}$ $\mathrm{L}_{9}$ para o caráter REND, nos dois locais. Essas linhagens com altos valores negativos terão médias de seus cruzamentos menores que a média geral dos híbridos simples. Desta forma, como exemplo, a linhagem $\mathrm{L}_{2}$ contribuirá com genes de ação aditiva que provocarão queda de rendimento de espigas nos cruzamentos em que participar (Vencovsky \& Barriga, 1992).

Altos valores de $\mathrm{S}_{\mathrm{ij}}$ (com sinal positivo ou negativo) indicam algumas combinações específicas que são melhores ou piores que o esperado, baseando-se na CGC dos genitores (Sprague \& Tatum, 1942). Os mesmos autores afirmaram que esses valores constituem uma indicação importante dos genes que exibem efeitos de epistasia e dominância.
As combinações que mostraram máximo efeito de CEC para PECP corresponderam a $\mathrm{L}_{1} \times \mathrm{L}_{5}$ e $\mathrm{L}_{1} \times \mathrm{L}_{8}$, com base no valor médio de $\mathrm{S}_{\mathrm{ij}}$ nos dois locais (Tabela 2). Considerando o caráter PESP, os cruzamentos de maiores valores foram $\mathrm{L}_{1} \times \mathrm{L}_{8} \mathrm{e} \mathrm{L}_{4} \times \mathrm{L}_{8}$ em Pesqueira e $\mathrm{L}_{1} \times \mathrm{L}_{9} \mathrm{e} \mathrm{L}_{1} \mathrm{x} \mathrm{L}_{3}$ em Vitória de Santo Antão. Para REND, os cruzamentos $\mathrm{L}_{4}$ x $\mathrm{L}_{8}$ e $\mathrm{L}_{8}$ x L $\mathrm{L}_{10}$ em Pesqueira $\mathrm{e}_{7} \mathrm{x} \mathrm{L}_{8} \mathrm{e}$ $\mathrm{L}_{5} \times \mathrm{L}_{9}$ em Vitória de Santo Antão foram os que apresentaram os maiores efeitos. Os cruzamentos com altos efeitos de CEC $\left(\mathrm{S}_{\mathrm{ij}}\right)$ envolveram, usualmente, um alto combinante geral como um genitor ou dois baixos combinantes gerais. Resultados semelhantes foram encontrados por Lopes et al. (1985).

Para o caráter PECP, os valores para heterose nos cruzamentos relativos à média dos pais (MP) variaram de $-26,43 \%$ a $117,51 \%$ em Pesqueira e de $-34,62 \%$ a $66,39 \%$ em Vitória de Santo Antão. Em relação ao pai mais produtivo (PMP) variaram de -36,96 a 88,20\% em Pesqueira e de $-39,81 \%$ a $32,57 \%$ em Vitória de Santo Antão. Os cruzamentos $\mathrm{L}_{1} \times \mathrm{L}_{8}$ e $\mathrm{L}_{1} \times \mathrm{L}_{3}$ foram os que apresentaram os maiores efeitos heteróticos para ambos MP e PMP em Pesqueira e Vitória de Santo Antão, respectivamente.

Considerando o caráter PESP, os efeitos heteróticos nos cruzamentos re- lativos à MP variaram de $-37,70 \%$ a $165,71 \%$ em Pesqueira e de $-25,52 \%$ a 94,40\% em Vitória de Santo Antão. Em relação ao PMP variaram de $-45,007 \%$ a $125,43 \%$ em Pesqueira e de $-36,56$ a $45,98 \%$ em Vitória de Santo Antão. Os cruzamentos $\mathrm{L}_{1} \times \mathrm{L}_{8}$ e $\mathrm{L}_{1} \times \mathrm{L}_{3}$ que se destacaram para o caráter PECP também apresentaram o mesmo desempenho para PESP com relação ao efeito heterótico.

Para o caráter REND, os valores de heterose nos cruzamentos relativos à MP variaram de $-20,19 \%$ a $24,01 \%$ em Pesqueira e de $-18,02 \%$ a $16,76 \%$ em Vitória de Santo Antão. Em relação ao PMP variaram de $-23,52$ a $22,99 \%$ em Pesqueira e de-22,05 a 12,03\% em Vitória de Santo Antão. $\mathrm{O}$ híbrido $\mathrm{L}_{1} \times \mathrm{L}_{8} \mathrm{se}$ destacou com maior efeito heterótico relativo à MP e PMP em Pesqueira. Em Vitória de Santo Antão se destacaram com maiores valores de MP e PMP, respectivamente os híbridos $\mathrm{L}_{1} \times \mathrm{L}_{7}$ e $\mathrm{L}_{7} \times \mathrm{L}_{8}$.

A característica mais importante para a indústria é sem dúvida o caráter REND, uma vez que a comercialização das espigas é realizada por unidade e com palha e índices inferiores a 50\% inviabilizam comercialmente o produto. Portanto, para o caráter, as linhagens $\mathrm{L}_{4}$, $\mathrm{L}_{5}$ e $\mathrm{L}_{7}$ apresentaram os maiores valores na estimativa da CGC, sendo as mais favoráveis em aumentar o caráter nos dois 
Tabela 2. Estimativas dos efeitos da capacidade específica de combinação $\left(\mathrm{S}_{\mathrm{ij}}\right)$, percentagem de heterose para os cruzamentos relativos à média dos pais (MP) e pai mais produtivo (PMP), médias de peso de espiga com palha (PECP), peso de espiga sem palha (PESP) e percentagem de rendimento de espiga (REND) para os cinco híbridos simples mais promissores. Pesqueira e Vitória de Santo Antão, UFRPE/IPA, 1996/97.

\begin{tabular}{|c|c|c|c|c|c|c|c|c|c|}
\hline \multirow{3}{*}{ Híbridos } & \multicolumn{4}{|c|}{ Pesqueira } & \multirow{3}{*}{ Híbridos } & \multicolumn{4}{|c|}{ Pesqueira } \\
\hline & \multicolumn{4}{|c|}{ Heterose (\%) } & & \multicolumn{4}{|c|}{ Heterose (\%) } \\
\hline & Sij & MP & PMP & (g/planta) & & Sij & MP & PMP & (g/planta) \\
\hline & \multicolumn{9}{|c|}{ PECP } \\
\hline $\mathrm{L}_{1} \mathrm{xL}_{5}$ & 81,814 & 76,61 & 74,86 & $370,0 a b$ & $\mathrm{~L}_{1} \mathrm{xL}_{9}$ & 84,149 & 34,60 & $-13,41$ & $494,8 a \ldots d$ \\
\hline $\mathrm{L}_{1} \mathrm{xL}_{8}$ & 78,681 & 117,51 & 83,79 & $388,9 a$ & $\mathrm{~L}_{1} \mathrm{xL}_{8}$ & 63,008 & 60,20 & 18,05 & $408,1 \mathrm{a} \ldots \mathrm{g}$ \\
\hline $\mathrm{L}_{1} \mathrm{xL}_{6}$ & 64,923 & 42,94 & 21,83 & $368,0 a b c$ & $\mathrm{~L}_{1} \mathrm{xL}_{4}$ & 58,616 & 58,67 & 19,32 & $387,8 a \ldots g$ \\
\hline $\mathrm{L}_{3} \mathrm{xL}_{8}$ & 59,073 & 108,55 & 88,20 & $341,4 a \ldots f$ & $\mathrm{~L}_{2} \mathrm{xL}_{6}$ & 56,624 & 21,78 & 10,58 & $513,0 \mathrm{abc}$ \\
\hline \multirow[t]{2}{*}{$\underline{\mathrm{L}_{3} \times \mathrm{L}_{6}}$} & 58,414 & 38,89 & 10,98 & $336,6 \mathrm{a} \ldots \mathrm{g}$ & $\mathrm{L}_{8} \times \mathrm{L}_{9}$ & 54,999 & 9,98 & $-11,74$ & $504,3 a b c$ \\
\hline & \multicolumn{9}{|c|}{ PESP } \\
\hline$\overline{L_{1} \times L_{8}}$ & 63,055 & 165,71 & 125,43 & $258,8 a b$ & $\mathrm{~L}_{1} \mathrm{xL}$ & 57,031 & 54,35 & $-1,90$ & $289,4 a \ldots d$ \\
\hline $\mathrm{L}_{4} \times \mathrm{L}_{8}$ & 62,913 & 82,82 & 27,03 & $260,8 a$ & $\mathrm{~L}_{1} \times \mathrm{L}_{3}$ & 44,989 & 94,40 & 45,98 & $232,7 a \ldots d$ \\
\hline $\mathrm{L}_{3} \mathrm{xL}_{6}$ & 59,005 & 53,53 & 18,57 & $245,8 a \ldots d$ & $\mathrm{~L}_{5} \mathrm{xL}_{7}$ & 40,948 & 13,33 & $-4,22$ & $319,6 a$ \\
\hline $\mathrm{L}_{1} \mathrm{xL}_{6}$ & 53,638 & 56,10 & 21,27 & $246,4 a b c$ & $\mathrm{~L}_{1} \times \mathrm{L}_{6}$ & 39,164 & 69,82 & 13,10 & $269,3 a \ldots d$ \\
\hline \multirow{2}{*}{$\mathrm{L} 8 \mathrm{xL}_{10}$} & 51,263 & 88,70 & 41,29 & $227,2 a \ldots f$ & $\mathrm{~L}_{1} \mathrm{XL}_{4}$ & 37,714 & 61,83 & 9,03 & $251,0 a \ldots d$ \\
\hline & \multicolumn{9}{|c|}{ REND(\%) } \\
\hline$\overline{\mathrm{L} \mathrm{xL}_{8}}$ & 0,086 & 20,26 & 7,30 & $74,95 a$ & $\mathrm{~L}_{7} \times \mathrm{L}_{8}$ & 0,071 & 14,73 & 12,03 & $70,33 a \ldots e$ \\
\hline $\mathrm{L} 8 \mathrm{xL}_{10}$ & 0,077 & 19,55 & 13,87 & 68,95a...f & $\mathrm{L}_{5} \mathrm{x} \mathrm{L}_{9}$ & 0,050 & 9,94 & $-0,34$ & 69,36a...f \\
\hline $\mathrm{L}_{3} \mathrm{xL}_{6}$ & 0,071 & 12,23 & 7,70 & $74,10 a b$ & $\mathrm{~L}_{1} \mathrm{xL}_{7}$ & 0,044 & 16,76 & 4,38 & $68,57 a \ldots f g$ \\
\hline$\underline{\mathrm{L}_{3} \times \mathrm{L}_{5}}$ & 0,070 & 6,70 & $-2,42$ & $74,45 \mathrm{ab}$ & $\mathrm{L}_{1} \times \mathrm{L}_{6}$ & 0,041 & 15,67 & 5,96 & $66,16 a \ldots$ \\
\hline $\mathrm{L}_{1} \times \mathrm{L}_{9}$ & 0,057 & 18,70 & 14,13 & 66,65a...i & $\mathrm{L}_{4} \mathrm{xL} \mathrm{L}_{8}$ & 0,035 & 5,70 & $-0,17$ & $70,55 a \ldots d$ \\
\hline
\end{tabular}

Médias seguidas de mesma letra nas colunas não diferem estatisticamente entre si a $5 \%$ de probabilidade pelo teste de Duncan

locais, enquanto que os híbridos apresentaram especificidade por local, sendo os cruzamentos $\mathrm{L}_{4} \times \mathrm{L}_{8}$ e $\mathrm{L}_{8} \times \mathrm{L}_{10}$ os mais promissores para Pesqueira e $\mathrm{L}_{7} \times \mathrm{L}_{8}$ e $\mathrm{L}_{5}$ $\mathrm{x} \mathrm{L}_{9}$ para Vitória de Santo Antão.

\section{LITERATURA CITADA}

ALLARD, R.W. Principles of plant breeding. John Wiley \& Sons, Inc, New York, 1960. 485 p. COCHRAN, W.G.; COX, C.M. Experimental designs. New York: John Wiley \& Sons, 1957. $611 \mathrm{p}$.

GNOATTO, I.L. Análise de cruzamentos dialélicos entre linhagens de milho (Zea mays L.) de diversas origens. Piracicaba: ESALQ, 1969. 80 p. (Tese mestrado).

GRIFFING, B. Concept of general and specific combining ability in relation to diallel crossing systems. Australian Journal of Biological Sciences, v. 9, n. 4, p. 463-493, 1956.
LIMA, M.; MIRANDA FILHO, J.B.; FURLANI, P.R. Diallel cross among inbred lines of maize differing in aluminium tolerance. Revista Brasileira de Genética, Ribeirão Preto, v. 18, n. 4, p. 579-584, 1995.

LOPES, M.A.; GAMA, E.E.G.; VIANNA, R.T.; SOUZA, I.R.P. Heterose e capacidade de combinação para produção de espigas em cruzamentos dialélicos de seis variedades de milho. Pesquisa Agropecuária Brasileira, Brasília, v. 20, n. 3, p. 349-354, 1985.

NASPOLINI FILHO, V.; GAMA, E.E.G.; VIANNA, R.T.; MORO, J.R. General and specific combining ability for yield in a diallel cross among 18 maize populations (Zea mays L.). Revista Brasileira de Genética, Ribeirão Preto, v. 4, n. 4, p. 571-577, 1981.

PARENTONI, S.N.; GAMA, E.E.G.; REIFSCHNEIDER, F.J.B.; GUIMARÃES, P.E.O. Avaliação da capacidade combinatória de dez linhagens de milho doce. Horticultura Brasileira, Brasília, v. 9, n. 2, p. 71-73, 1991.
SOUZA, I.R.P.; MAIA, A.H.N.; ANDRADE, C.L.T. Introdução e avaliação de milho doce na região do baixo Parnaíba. Parnaíba: EMBRAPACNPAI, 1990. 7 p. (Pesquisa em Andamento, 3). SPRAGUE, G.F.; TATUM, L.A. General vs. specific combining ability in single crosses of corn. Journal of the American Society of Agronomy, v. 34, n. 10, p. 923-932, 1942.

TRACY, W.F. Sweet corn. In: Hallauer, A. R. Specialty corns. New York, CRC Press, Boca Raton, 1994. p. 148-187.

VENCOVSKY, R. Análise de cruzamentos dialélicos entre variedades pelo método de Gardner e Eberhart. Piracicaba, ESALQ 1969, p. 99-111 (Relatório do Instituto de Genética).

VENCOSVSKY, R.; BARRIGA, P. Genética biométrica no fitomelhoramento. Ribeirão Preto, Revista Brasileira de Genética, 1992. 486 p.

WALLACE, H.A.; BRESSMAN, E.N. Classification of corn. In: WALLACE, H.A. \& BRESSMAN, E.N. ed. Corn and corn growing. New York, John Wiley \& Sons Inc., Chapman \& Hall Ltd., 1949. p. 52-60. 